Review

\title{
Synthesis and Neurotoxicity of Tetrahydroisoquinoline Derivatives for Studying Parkinson's Disease
}

\author{
Kenji Abe, ${ }^{a}$ Toshiaki Saitoh, ${ }^{b}$ Yoshie Horiguchi, ${ }^{b}$ Iku UtsunomiYa, ${ }^{a}$ and Kyoji Taguchi ${ }^{*}, a$ \\ ${ }^{a}$ Department of Neuroscience, Showa Pharmaceutical University; and ${ }^{b}$ Department of Medicinal Chemistry, Showa Pharmaceutical \\ University; 3-3165 Higashitamagawagakuen, Machida, Tokyo 194-0042, Japan.
}

Received February 4, 2005; accepted May 6, 2005

\begin{abstract}
Parkinson's disease involves the progressive degeneration of dopaminergic neurons in the substantia nigra. However, the etiology of the disease remains to be elucidated. Endogenous amines, such as 1,2,3,4-tetrahydroisoquinoline (TIQ) derivatives present in the mammalian brain, are known to participate in the pathogenesis of Parkinson's disease. These endogenous neurotoxins have been extensively studied because of their structural resemblance to 1-methyl-4-phenyl-1,2,3,6-tetrahydropyridine (MPTP), an agent widely used for generating animal models of Parkinson's disease-like symptoms. Investigations of the synthesis and pharmacological properties of TIQ derivatives are expected to contribute to the development of new therapeutic agents for treating Parkinson's disease. In the present study, we describe more efficient synthesis methods for TIQ derivatives via Pummerertype cyclization of the substrate $N$-acyl sulfoxide. Furthermore, the modified Pummerer reaction provided a convenient and efficient method for synthesizing various TIQs. TIQ and its derivative, 1-benzyl-TIQ, can induce parkinsonism in primates and rodents. On the other hand, one TIQ derivative, 1-methyl-TIQ, has been shown to prevent MPTP, TIQ, and 1-benzyl-TIQ induced behavioral abnormalities. Therefore, TIQ derivatives are considered to play an important role in both the onset and prevention of Parkinson's disease. In this article, we focus on the synthesis and pharmacological aspects of 1,2,3,4-tetrahydroisoquinoline derivatives in Parkinson's disease.
\end{abstract}

Key words Parkinson's disease; Pummerer type cyclization; 1,2,3,4-tetrahydroisoquinoline; 1-benzyl-1,2,3,4-tetrahydroisoquinoline; 1-methyl-1,2,3,4-tetrahydroisoquinoline

\section{INTRODUCTION}

Parkinson's disease (PD) is a neurodegenerative disorder characterized by bradykinesia, rigidity and tremors. Pathological changes associated with PD include death of dopaminergic neurons in the substantia nigra pars compacta and Lewy bodies in some of the surviving neurons. While the causes of slow neuronal death in PD are unknown, they probably involve genetic and environmental factors. Recently, the gene responsible for familial PD was identified, ${ }^{1,2)}$ enabling etiological investigations of familial PD to progress rapidly. However, familial PD represents only a fraction of PD, and less information is available on the etiology of idiopathic PD. Thus, a genetic predisposition toward PD is suspected, although environmental factors are believed to play a major role. For example, 1-methyl-4-phenyl-1,2,3,6-tetrahydropyridine (MPTP) is a very selective neurotoxin that produces a clinical syndrome similar to PD. ${ }^{3)}$ MPTP, furthermore, exerts its effects on nigrostriatal dopaminergic neurons in several animal species, including human, ${ }^{3,4)}$ monkey, ${ }^{5)} \mathrm{cat}^{6}, \mathrm{rat}^{7)}$ and mouse. ${ }^{8)}$ Therefore, MPTP forms the basis for studying the role of non-genetic factors associated with PD.

Since MPTP is an exogenous compound, other endogenous toxins may also accumulate in the central nervous system and cause PD. Hao et al. reported that a low molecular weight fraction (less than 10000) from PD cerebro-spinal fluid was cytotoxic to mesencephalon cells in vitro. ${ }^{9)}$ Moreover, cytotoxicity was reduced by deprenyl, a therapeutic drug for PD. ${ }^{10)}$ These results suggest that substances present in cerebro-spinal fluid caused PD. Isoquinoline derivatives are of interest as possible neurotoxins because they share structural similarity with MPTP and its active metabolite, 1methyl-4-phenylpyridinium ion $\left(\mathrm{MPP}^{+}\right)$. These compounds are synthesized in vivo by Pictet-Spengler condensation of biogenic amines with aldehydes. Metabolism occurs through cytochrome $\mathrm{P} 450, N$-methyltransferase and monoamine oxidase (MAO)-B to produce $N$-methylated isoquinoline and $N$ methylisoquinolinium, ${ }^{11-13)}$ which leads to charged quaternary forms (isoquinolinium cations). ${ }^{14-16)}$ Isoquinoline derivatives have been extensively studied because of their structural resemblance to MPTP, an agent widely used for generating animal models of PD. ${ }^{17}$

In the present report, we review the chemical synthetic methods for tetrahydroisoquinoline derivatives and their neurotoxic effects on the nigrostriatal dopaminergic system. It is hoped that this information may be useful for the treatment of basal ganglia disorders, such as PD.

\section{SYNTHESIS OF TETRAHYDROISOQUINOLINE DERIVATIVES}

Endogenous isoquinolines, such as 1,2,3,4-tetrahydroisoquinoline (TIQ), present in the human brain were previously reported to participate in the pathogenesis of PD. ${ }^{18-21)} \mathrm{TIQ}$ derivatives (TIQs) have also been found in the brain of other animal species, ${ }^{19,22,23)}$ and can induce parkinsonism in primates and rodents. ${ }^{24,25)}$ TIQ is commonly found in a variety of foods (e.g. milk, cocoa and bananas) ${ }^{26,27)}$ and it easily penetrates the blood-brain barrier. ${ }^{28,29)} \mathrm{N}$-Methylation of TIQ and oxidation of $N$-methylated TIQ has been shown to increase neurotoxicity. ${ }^{12,13)}$

Methods for synthesizing the isoquinoline ring system include the Pictet-Spengler reaction, ${ }^{30)}$ the Bischler-Napieralski reaction, ${ }^{31)}$ and the Pomeranz-Fritsch reaction. ${ }^{32,33)}$ Of these reactions, the Pictet-Spengler reaction and the Bischler-Napieralski reaction have proven effective for synthesizing TIQs and 1,2-dihydroisoquinoliones. In fact, 1-alkyl6,7-dihydroxy-TIQs (4) were prepared by the Bischler- 


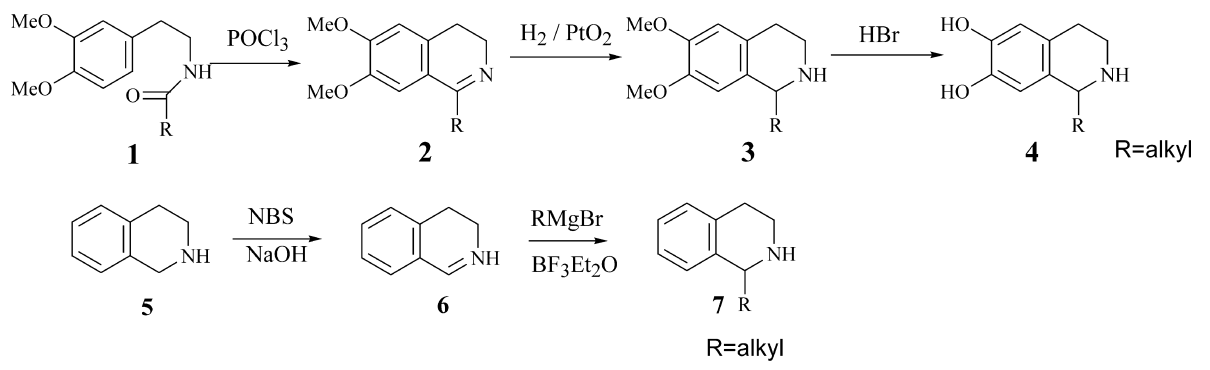

Chart 1

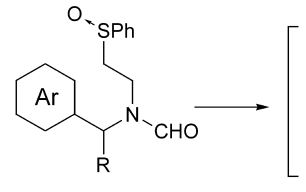

8

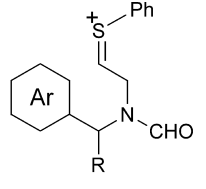

9

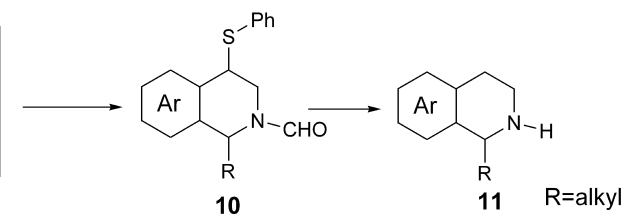

Chart 2
Napieralski reaction of amides of 3,4-dimethoxyphenylethylamine, catalytic reduction of the obtained dihydroisoquinolines (2) followed by demethylation of the resulting 1-alkyl6,7-dimethoxy-TIQs (3) with hydrobromic acid (Chart 1). ${ }^{34)}$ Several publications have reported the preparation of 1-substituted TIQs via the Pictet-Spengler reaction or BischlerNapieralski reaction. ${ }^{35-37)}$ Venkov demonstrated the synthesis of 1-substituted $N$-acyl-TIQs from $N$-acyl-2-phenylethylamines and aldehyde catalyzed by acidic media. ${ }^{38)}$ Other reports of substituted TIQ synthesis include Kametani's phenolic cyclization of 3-hydroxyphenylethylamine with carbonyl compounds. ${ }^{39-41)}$ Although there are effective methods for constructing the isoquinoline ring via the Pictet-Spengler reaction or the Bischler-Napieralski reaction, it is still difficult to synthesize TIQs that lack electron-donating groups in the aromatic ring. Generally, preparation of 1-alkyl-TIQs (7) can be achieved by nucleophilic addition ${ }^{42-45)}$ using organometallic reagents and 3,4-dihydroisoquinoline (6) derived from commercially available TIQ (5) as shown in Chart $\left.1 .{ }^{46}\right)$

Recently, Yokoyama and coworkers reported the use of the super acid-catalyzed Pictet-Spengler reaction for the synthesis of 1-substituted TIQs. This cyclization reaction of intermediate imines of 2-arylethylamine was accelerated by the presence of super acid, trifluoromethane sulfonic acid (TFSA). ${ }^{47)}$ Furthermore, Takano et al. reported the synthesis of TIQs using sulfoxide-mediated electrophillic cyclization (Pummerer reaction) as a key step. ${ }^{48}$ Our group modified Takano's synthesis and discovered a highly efficient method for preparing TIQs, with excellent overall yields, starting from aromatic aldehydes, aromatic ketones, and arylmethylamines, as shown in Chart 2. ${ }^{49-53)}$

$N$-Formyl sulfoxides (15), the substrates for the Pummerer reaction, were prepared from aromatic aldehyde (12) and 2phenylsulfanylethylamine (13). Condensation of 12 and 13 in EtOH followed by sodium borohydride reduction of resulting imines gave $N$-(aryl)methyl-2-(phenylsulfanyl)ethylamines (14). The amine (14) was protected by formylation and oxidized with sodium metaperiodate $\left(\mathrm{NaIO}_{4}\right)$ to give sulfoxide (15) in high yields. The Pummerer reaction of sulfoxide (15a) proceeded under a mild condition with treatment of trifluoroacetic anhydride (TFAA) in benzene at room temperature, and the cyclization reaction readily proceeded to produce the $N$-formyl-4-phenylsulfanyl TIQ (16a) in quantitative yield.
On the other hand, the Pummerer reaction of the sulfoxide (15b) without an electron-donating group in the aromatic ring merely yielded a characterizable product. The Pummerer cyclization of this sulfoxide was achieved by using borontrifluoride diethyl etherate $\left(\mathrm{BF}_{3} \cdot \mathrm{Et}_{2} \mathrm{O}\right)$ as an additive acid during the cyclization reaction. This reaction produced the expected $N$ formyl-4-phenylsulfanyl-TIQ (16b) at a quantitative yield. This effect of $\mathrm{BF}_{3} \cdot \mathrm{Et}_{2} \mathrm{O}$ as an additive acid on this cyclization, although difficult to explain, may be attributable to enhancement of the electrophilic reactivity of the sulfonium cation, a key intermediate of this cyclization, by a change in the counter anion from $\mathrm{CF}_{3} \mathrm{COO}^{-}$to $\mathrm{CF}_{3} \mathrm{COO}-\mathrm{BF}_{3}^{-}$. We designated this reaction using TFAA and $\mathrm{BF}_{3} \cdot \mathrm{Et}_{2} \mathrm{O}$ as the cyclization reagents as the modified Pummerer reaction.

The conversion of $N$-formyl-4-phenylsulfanyl-TIQs (16) to the corresponding TIQs was readily achieved by conventional methods. Reductive removal of the phenylsulfanyl group of 16, which readily proceeded after treatment with $\mathrm{NiCl}_{2}-\mathrm{NaBH}_{4}$, produced $N$-formyl-TIQs (17). Alkaline hydrolysis or lithium aluminum hydride $\left(\mathrm{LiAlH}_{4}\right)$ reduction of 17 produced TIQs (18) and $N$-methyl-TIQs (19) at good yields, respectively (Chart 3). In the investigation, we identified two methods for the cyclization of sulfoxide. The reaction using TFAA in benzene at room temperature (method A) was effective only for the synthesis of TIQs with ortho or para methoxy groups relative to the cyclization position of the phenyl ring. In this ring system, the nucleophilic center of the phenyl ring was electronically activated. The alternative method, which involved sequential treatment with TFAA and $\mathrm{BF}_{3} \cdot \mathrm{Et}_{2} \mathrm{O}$ (method $\mathrm{B}$ : modified Pummerer reaction), was efficient for the preparation of TIQs that do not have an activated phenyl ring.

The application of the modified Pummerer reaction to the sulfoxide (22) provided a way to synthesize 1-alkyl-TIQs that lacked electron-donating groups in the aromatic ring. The sulfoxide (22) was prepared from phenylketones (20) and 13 by an imination reaction catalyzed by titanium(IV) isopropoxide followed by reduction, formylation and oxidation. 1-Methyl-TIQ (25a), 1-benzyl-TIQ (25b), and their $N$-methyl derivatives (26a, b) were prepared in seven steps from ketones (20) via the modified Pummerer reaction at overall yields of $55-68 \%$ (Chart 4 ). Thus, we demonstrated that the modified Pummerer reaction provided a convenient and effi- 


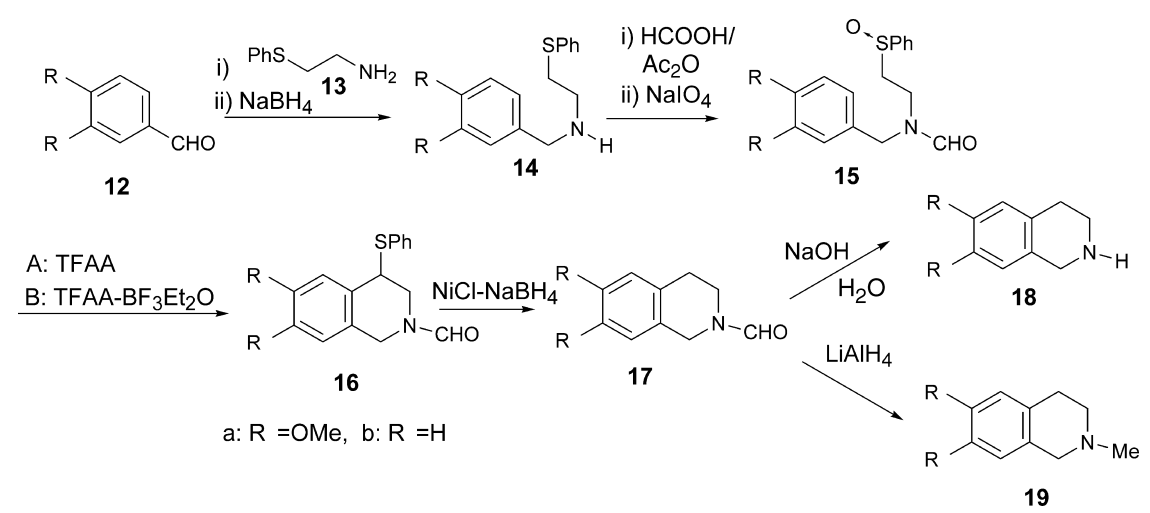

Chart 3
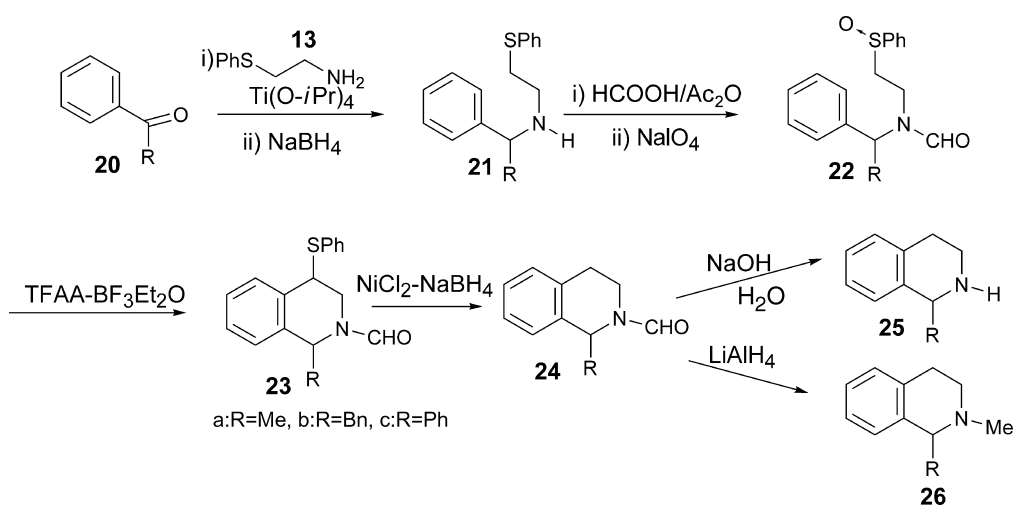

Chart 4

cient method for synthesizing various TIQs. 1-Benzyl-TIQ (25b) is produced by condensation and cyclization of two endogenous compounds, 2-phenylethylamine and phenylacetaldehyde, a metabolite of 2-phenylethylamine generated by MAO-B. 1-Benzyl-TIQ (25b) derivatives occur naturally in animal brain tissue, and they induce parkinsonism in monkeys and mice. ${ }^{19,54)}$

\section{SYNTHESIS OF CHIRAL TERTRAHYDROISO- QUINOLINE DERIVATIVES}

The endogenous amine, 1-methyl-TIQ, is enzymatically synthesized from 2-phenylethylamine and pyruvate in rat brain. ${ }^{21)} 1$-Methyl-TIQ is present in human, rat and mouse brains $^{17,22,24,55)}$ as a mixture of $(R)$ - and $(S)$-enantiomers. Its concentration decreases in the parkinsonian brain ${ }^{56}$ and during aging, especially in the substantia nigra and striatum, which are implicated in $\mathrm{PD}^{57)} \mathrm{A}$ number of methods for synthesizing chiral TIQs have been reported. The key problem in the synthesis is the asymmetric introduction of the alkyl substituents at the $\mathrm{C}-1$ position of the tetrahydroisoquinoline nucleus. Meyers et al. reported efficient diastereoselective alkylation of 1-lithiated TIQ containing the formamidine auxiliary, ${ }^{52,53)}$ whereas Gawley demonstrated the stereoselective alkylation of chiral lithiated (tetrahydroisoquinolyl) oxasolines. ${ }^{58)}$ Other asymmetric syntheses of chiral 1-alkyl TIQs via the stereoselective alkylation of oxazolo[2,3-a]tetrahydroisoquinolines ${ }^{59,60)}$ or $N$-acylated tetrahydroisoquinolines using organometallic reagents have also been reported. ${ }^{61,62)}$ However, effective methods for efficiently synthesizing these compounds are still needed. The modified Pummerer reaction provides a method for synthesizing chiral 1-methyl, 1- benzyl and 1,3-dimethyl-TIQs. ${ }^{63)}$ The chiral sulfoxides $(29 a-d)$ were prepared in three steps from readily available chiral amines, $(R)$ - and $(S)$-1-alkyl phenylethylamine (27). The modified Pummerer reaction of sulfoxide (29) gave excellent yields of 1-alkyl-TIQs. Thus, optically pure $(R)$ - and $(S)$-1-methyl-TIQs (32a, b) and $(R)$ - and $(S)$-1,2-dimethylTIQs $(33 \mathbf{a}, \mathbf{b})$ were prepared from the starting material amine $(27 \mathbf{a}, \mathbf{b})$ at approximately $50 \%$ overall yield. Similarly, optically pure $(R)$ - and $(S)$-1-benzyl-TIQs $(\mathbf{3 2 c}, \mathbf{d})$ and $(R)$ - and $(S)$-1-benzyl-2-methyl-TIQs (33c, d) were prepared from $\mathbf{2 7 c}, \mathbf{d}$ at approximately $35 \%$ overall yield (Chart 5). Furthermore, four stereoisomers, 1,3-dimethyl-TIQs, were synthesized by applying the modified Pummerer reaction as the key step. ${ }^{64)}$ The four key intermediate chiral sulfides (38) were prepared in a stereochemically unambiguous manner via two routes from alaninol (route I) and 1-phenylethylamine (35) (route II). Reductive amination of acetophenone with the $(R)$ amine (34) prepared from $(R)$-alaninol yielded the two diastereomeric mixtures of sulfide, and the separation of the mixtures by MPLC gave the optically pure sulfides (36) and (37) at high yield. Similarly, enantiomers, (ent-36) and (ent37), were also prepared from $(S)$-amine (Chart 6).

Reductive amination of $(R)$-1-phenylethylamine (35) with phenylsulfanylacetone gave two diastereomeric mixtures of sulfide (36) and (ent-37). The mixture of sulfoxide (37) and (ent-36) was synthesized from $(S)$ 1-phenyletylamine (ent35). The modified Pummerer reaction of the corresponding sulfoxide (38a) of $\mathbf{3 6}$ gave the desired 4-phenylsulfanyl-TIQ (39a) at a moderate yield. Reductive removal of the phenylsulfanyl group followed by deprotection of the $N$-formyl group produced TIQ (40a). Other stereoisomeric TIQs (ent40a, 40b and ent-40b) were synthesized by the same proce- 

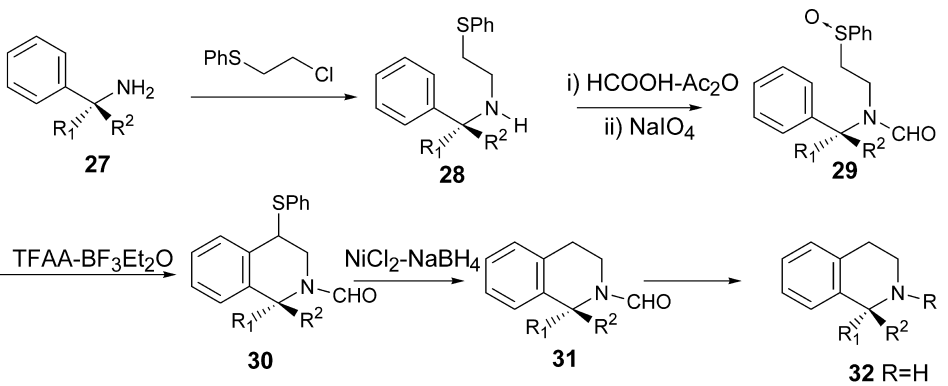

a: $R^{1}=M e, R^{2}=H, b: R^{1}=H, R^{2}=M e, c: R^{1}=B n, R^{2}=H, d: R^{1}=H, R^{2}=B n \quad 33 R=M e$

Chart 5

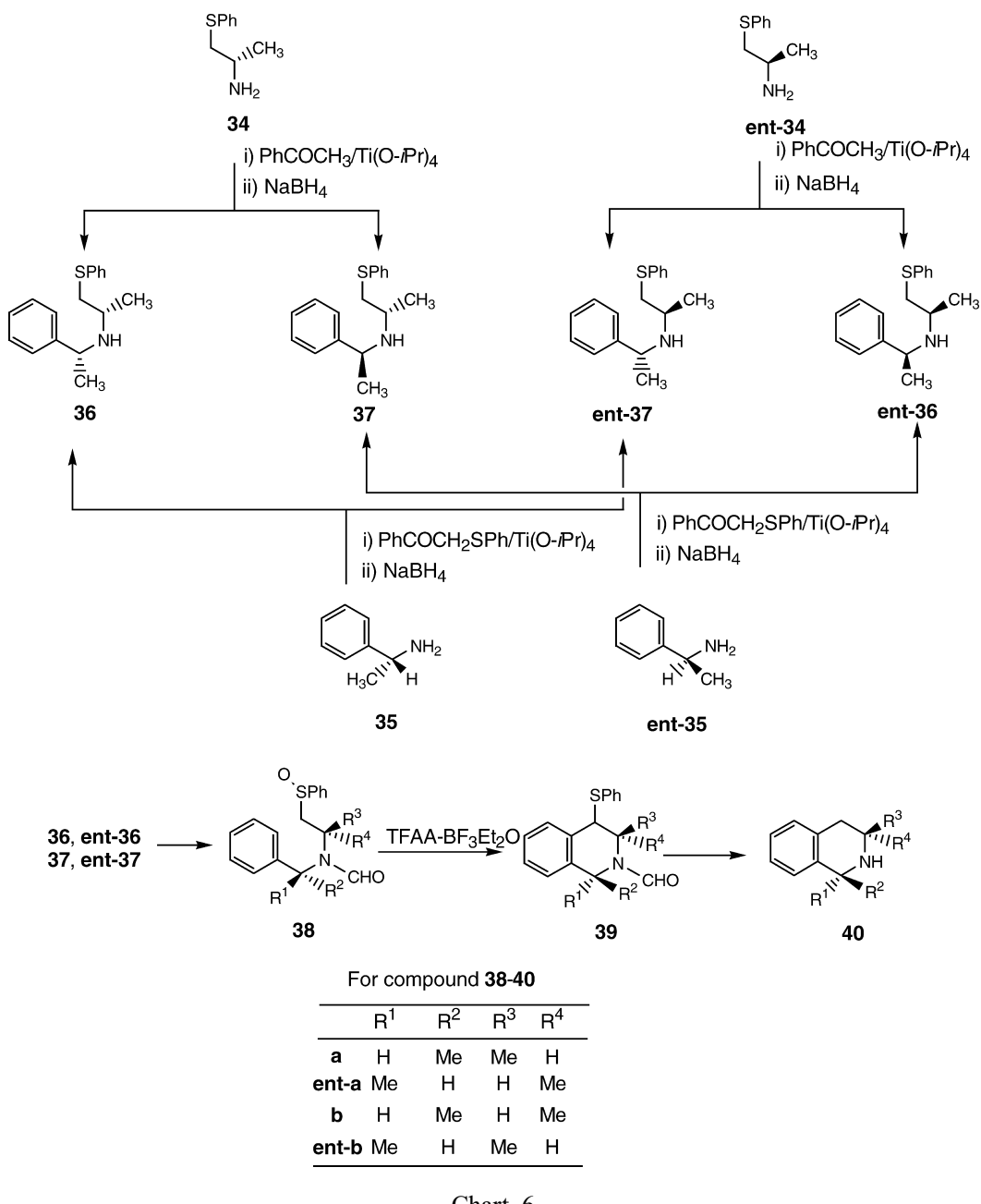

Chart 6

dure. Improvements in the modified Pummerer reaction that used TFSA instead of $\mathrm{BF}_{3} \cdot \mathrm{Et}_{2} \mathrm{O}$ as the additive acid resulted in an effective method for synthesizing four chiral 1,3-dimethyl-TIQs in satisfactory yield from the starting amines (35 and ent-35, Chart 6) ${ }^{65}$ The use of TFSA dramatically enhanced the cationic cyclization in the Pummerer reaction, suggesting that the reaction can involve a dicationic intermediate just like the super acid-catalyzed Pictet-Spengler reaction reported by Yokoyama et al. ${ }^{47)} \mathrm{A}$ detailed mechanistic study of Pummerer type cyclization reaction is in progress.

\section{NEUROTOXICITY OF 1,2,3,4-TETRAHYDROISO- QUINOLINE (TIQ)}

TIQ is activated to form neurotoxic $N$-methylated tetrahy- droisoquinolinium cations through $\mathrm{N}$-methylation by $\mathrm{N}$ methyltransferase and oxidation via MAO. ${ }^{12,13)}$ TIQ initiates degeneration of striatal dopaminergic neurons in the brains of chronically treated rats, which have been used as animal models for PD. ${ }^{66,67)}$ The chronic treatment of primates with TIQ produces parkinsonism, with reduced dopamine (DA), tyrosine hydroxylase (TH) and biopterine in the nigrostriatal region. ${ }^{68)}$ Chronic treatment with TIQ also reduces the number of TH-positive neurons in the substantia nigra pars compacta of mice and monkeys ${ }^{25,69)}$ and significantly decreases the number of TH-positive neurons in the substantia nigra and levels of DA and its metabolites in the rat striatum. ${ }^{66,67)}$ Although DA and its metabolites decrease in the striatum, no changes in DA have been observed in the substantia nigra and nucleus accumbens. ${ }^{66)}$ In contrast, a single dose of TIQ 
via microdialysis increases the DA levels in the rat striatum. ${ }^{67)}$ Booth et al. found that the local application of TIQ via a microdialysis probe also caused an increase in DA release in the striatum. ${ }^{70)}$ Thus, these results suggest that acute or short-term treatment with TIQ temporarily increases DA levels in the brain, while chronic treatment causes the levels to decline. Our previous study also showed that repeated treatment with TIQ decreased the DA content of the striatum and induced a loss of TH-positive cells in the substantia nigra of mice. ${ }^{71)}$ These biochemical studies indicated that TIQ damaged the nigrostriatal pathway and either facilitated or inhibited DA release in vivo. In addition, our data showed that doses producing behavioral abnormality did not reduce ligand and DA transporter (DAT) binding in the striatum of TIQ treated mice. This was not observed upon treatment with MPTP. ${ }^{72)}$ However, significant decreases in ligand-DAT binding were observed after larger doses of TIQ or longer treatment periods. ${ }^{72,73)}$

Although the process involved in the degradation of dopaminergic neurons in PD is not clear, DAT proteins may be responsible for the selectivity of dopaminergic cell death in this disease. ${ }^{74,75)}$ A reduced energy supply caused by inhibition of mitochondrial enzymes is also an important factor leading to cell death. ${ }^{76,77)}$ While TIQ inhibited the activity of complex I, it had no effect on complex II, III, or IV in mouse brain mitochondrial fragments. ${ }^{78,79)}$ The inhibition of mitochondrial respiration and disruption of mitochondrial function by TIQ appears to be related to dopaminergic cell death. The neurotoxins that are transported into the cell by DAT play an important role in mitochondrial enzyme activity. Thus, a relatively short-term treatment with TIQ temporarily stimulated dopaminergic neurons and then inhibited TH protein after increased DA release, without decreasing ligandDAT binding.

PD develops slowly during aging, which may provide the opportunity for long-term exposure and accumulation of substances that cause PD. Acute administration of TIQ may induce behavioral effects due to decreased DA and inhibition of TH protein, but does not produce cell death. This explains why reduced DA and TH-positive cells are not accompanied by decreasing ligand-DAT binding. Long-term treatment with TIQ causes cell death via mitochondrial dysfunction after DAT is concentrated in dopaminergic neurons. Thus, endogenous TIQ may participate in the degeneration of the nigrostriatal dopaminergic system in PD. These results suggest that high concentration and/or prolonged exposure to endogenous TIQ derivatives may cause degeneration of the nigrostriatal dopaminergic system in PD. It is important to determine whether TIQ-induced PD-like symptoms are caused physiologically by metabolized TIQ, such as $N$-methylated TIQ. However, the precise mechanism and location by which $N$-methylated TIQ induces PD remains unclear.

5. NEUROPROTECTION OF 1-METHYL-1,2,3,4TETRAHYDROISOQUINOLINE (1-METHYL-TIQ)

The levels of 1-methyl-TIQ are markedly depleted in the brains of patients with $\mathrm{PD},{ }^{56)}$ and endogenous levels of 1methyl-TIQ are reduced in brains of mice treated with MPTP. $^{24)}$ 1-Methyl-TIQ prevents MPTP-, TIQ- and 1-benzylTIQ-induced behavioral abnormalities, ${ }^{19,24)}$ and it is the only endogenous parkinsonism-preventing agent discovered to date. The inhibitory effect on mitochondrial respiration rate induced by $\mathrm{MPP}^{+}$that was seen in rat liver mitochondria and striatal synaptosomes was prevented by 1 -methyl-TIQ. ${ }^{80)}$ It was ascertained that $\mathrm{MPP}^{+}$exerts its toxicity by accumulation at catecholaminergic neurons and terminals via the catecholamine uptake sites. ${ }^{81)}$ Therefore, the protective effects of 1-methyl-TIQ are due to the inhibition of $\mathrm{MPP}^{+}$uptake into the striatal synaptosome. Furthermore, 1-methyl-TIQ strongly inhibits DA MAO-B-dependent $\mathrm{N}$-oxidation and increases COMT-dependent $O$-methylation two-fold. An effect on DA catabolism may reduce the generation of free radicals, and protect dopaminergic neurons. ${ }^{82)}$ However, 1-methyl-TIQ showed no affinity for DA receptors and did not directly prevent the inhibition of mitochondrial respiratory complex I in cultured rat mesencephalic neurons by $\mathrm{MPP}^{+}$or 1-benzylTIQ ${ }^{83)}$ It was hypothesized that 1-methyl-TIQ acts as an anti-oxidant during the induction of anti-oxidative enzymes. More direct evidence will be provided by future studies.

Our previous studies showed that 1-methyl-TIQ inhibited TIQ-induced bradykinesia. ${ }^{71}$ We also investigated the stereoisomer effects of 1-methyl-TIQ on TIQ-induced parkinsonism using $(R)$ - and $(S)$-enantiomers. Our results showed that $(R)$-1-methyl-TIQ was a potent inhibitor of TIQinduced bradykinesia. Moreover, $(R)$-1-methyl-TIQ prevented the loss of TH-positive cells in the substantia nigra pars compacta and decreased DA in the striatum compared to $(S)$-1-methyl-TIQ. ${ }^{71)}$ Recently, $(R)$-1-methyl-TIQ was found to exert a dose-dependent neuroprotective effect on physiological neuronal cell death, while $(S)$-1-methyl-TIQ had little effect. ${ }^{83)}$ From these results, we initially thought that $(R)-1$ methyl-TIQ played a crucial role in TIQ-induced PD-like symptoms. However, additional studies using in vivo binding of radioligand to presynaptic DAT or DA $\mathrm{D}_{2}$ receptors showed that $(S)$-1-methyl-TIQ had a protective effect on MPTP-induced loss of ligand-DAT binding that was more potent than $(R)-1$-methyl-TIQ in the mouse striatum. ${ }^{72)}(R)$ 1-Methyl-TIQ had only a weak protective effect on MPTPinduced loss of ligand-DAT binding. Moreover, although $(R)-$ 1-methyl-TIQ had only a very weak protective effect against TIQ-induced lowered ligand-DAT binding, $(S)$-1-methyl-TIQ had a potent effect on reduced ligand-DAT binding. ${ }^{72)}$ These findings suggest that both $(R)$ - and $(S)$-enantiomers may be endogenous agents that prevent PD by different mechanisms.

These observations support the hypothesis that both $(R)$ and $(S)$-1-methyl-TIQ play a crucial role in the protection of MPTP- and TIQ-induced parkinsonism. A change in enantiometric 1-methyl-TIQ biosynthesis, such as an unusual enantiometric ratio of 1-methyl-TIQ being produced (usually, the production ratio of $(R)$ - and $(S)$-1-methyl-TIQ is $6: 10$ in the mouse brain $)^{55)}$ may participate in the pathogenesis of PD. However, it cannot explain why enantiometric 1-methyl-TIQ produced different effects. Thus, it will be important to investigate the role of the $\mathrm{C}$ - 1 chiral center (absolute configuration) of 1-methyl-TIQ.

\section{NEUROTOXICITY OF 1-BENZYL-1,2,3,4-TETRA- HYDROISOQUINOLINE (1-BENZYL-TIQ)}

Chronic administration of 1-benzyl-TIQ induced parkinsonism in primates, and it may be related to idiopathic PD. ${ }^{54)}$ 
1-Benzyl-TIQ is a neurotoxin that causes parkinsonism in humans, and it is present in the cerebro-spinal fluid of parkinsonian patients at about three times the concentration found in healthy individuals. ${ }^{19)}$ In addition, L-deprenyl, a drug used in the treatment of PD, decreased endogenous levels of 1-benzyl-TIQ in mouse brains. ${ }^{84)}$ AntkiewiczMichaluk et al. reported that 1-benzyl-TIQ decreased striatal DA levels in rats up to $60 \%$ and increased levels of its metabolite, homovanillic acid, up to $40 \%$ relative to controls. $^{82)}$ Furthermore, 1-benzyl-TIQ significantly increased the rate of DA metabolism by the oxidative MAO-Bdependent catabolic pathway to mainly 3,4-dehydroxyphenylacetic acid (DOPAC) ${ }^{82}$ ) 1-Benzyl-TIQ has an affinity for the phencyclidine receptor, and it induced a stereotyped behavior and ataxia. ${ }^{84)}$ Simultaneous addition of NMDA receptor antagonist AP-5 to cultured slices does not affect the decrease in DA content caused by 1-benzyl-TIQ ${ }^{86)}$ which suggests that 1-benzyl-TIQ does not exert its toxic action via the NMDA receptor.

On the other hand, our data demonstrated that short term administration of 1-benzyl-TIQ did not induce the loss of TH-positive cells in spite of its bradykinesia-inducing effect and increased striatal DA. ${ }^{87)}$ In addition, 1-benzyl-TIQ profoundly stimulated DA release, which resulted in the competitive inhibition of $\left[{ }^{11} \mathrm{C}\right]$ raclopride and $\mathrm{DA} \mathrm{D}_{2}$ receptor binding, but not the induction of receptor degeneration. ${ }^{88)} \mathrm{Re}$ cently, we confirmed by microdialysis technique that systemic single administration of 1-benzyl-TIQ increased striatal DA content and spontaneous activity in a dose-dependent manner in rats (unpublished data). Thus, increased striatal DA content from a single or short-term treatment with 1benzyl-TIQ induced a behavioral abnormality. Furthermore, a dose of 1-benzyl-TIQ that produced bradykinesia did not reduce the in vivo specific binding of ligands to presynaptic DAT in the mouse striatum. ${ }^{72)}$ In addition, prolonged administration of 1-benzyl-TIQ also did not reduce ligand-DAT binding in the striatum, in contrast to TIQ. ${ }^{73)}$

As mentioned above, since PD develops slowly over time, there may be a long-term exposure or accumulation of weak PD-causing substances. 1-Benzyl-TIQ may need a longer time period than TIQ before symptoms, such as decrease in TH-positive cells or ligand-DAT binding, become evident. Increased levels of DA induced by 1-benzyl-TIQ are catabolized primarily by 1-benzyl-TIQ to DOPAC in dopaminergic terminals. DOPAC leads to the formation of free radicals that can damage dopaminergic neurons and lead to the neurotoxic effects associated with 1-benzyl-TIQ. The free radicals may result in a toxic difference between TIQ and 1-benzyl-TIQ. Non-selective neurotoxicity of free radicals may delay specific biochemical degeneration of the nigrostriatal dopaminergic system.

In contrast to relatively mild effects in vivo, 1-benzyl-TIQ has more potent cytotoxicity than $\mathrm{MPP}^{+}$in vitro. In this regard, 1-benzyl-TIQ inhibits mitochondrial respiration chain complex I or the apoptotic mechanism that increases proapoptotic protein and decreases anti-apoptotic protein. ${ }^{89-91)}$ In addition, 1-benzyl-TIQ decreases DA content and the number of TH-positive cells using the mesencephalon slice culture. An explanation for the difference in potency between in vivo and in vitro is still not clear. Investigations concerning active metabolites or active analogues of 1-benzyl-TIQ and detailed pharmacological studies are necessary to understand the mechanisms of action for 1-benzyl-TIQ. Long-term administration of 1-benzyl-TIQ may be required to produce degeneration of nigrostriatal dopaminergic neurons.

A derivative of 1-benzyl-TIQ, 1-(3',4'-dihydroxybenzyl)TIQ, is also an endogenous parkinsonism-inducing amine in animals. ${ }^{19,54)}$ Recently, Storch et al. reported that $2[N]$ methylated isoquinoline derivatives were selectively toxic to dopaminergic neurons via uptake by the DAT, and they may play a role in the pathogenesis of $\mathrm{PD}^{92)}$ On the other hand, Okuda et al. investigated the effects of various neuroprotective 1-methyl-TIQ derivatives on salsolinol induced cell death in vitro, and they reported hydroxymethylated 1methyl-TIQ had more potential for treating PD. ${ }^{93)}$ We believe that more toxic or more neuroprotective TIQ derivatives will be found.

References for the leading TIQ derivatives discussed in this review are summarized in Table 1 . Several lines of evidence suggest the dopaminergic neurons are involved in TIQ deriva-

Table 1. References of Typical TIQ Derivatives

\begin{tabular}{|c|c|c|c|c|}
\hline \multirow{2}{*}{ Name of compound } & \multirow{2}{*}{ Chemical structure } & \multicolumn{3}{|c|}{ References } \\
\hline & & Chemical synthesis & Biological synthesis & Pharmacological aspects \\
\hline TIQ & & $30-33$ & - & $12,13,18-29,66-82$ \\
\hline 1-Methyl-TIQ & & $\begin{array}{l}35-51 \text { (racemate) } \\
52,53,58-64 \text { (chiral) }\end{array}$ & 21 & $\begin{array}{l}17,19,22,24,25,55-57, \\
76,77,83\end{array}$ \\
\hline 1-Benzyl-TIQ & & $\begin{array}{l}35-51 \text { (racemate) } \\
52,53,58-64 \text { (chiral) }\end{array}$ & - & $19,77,78,84-94$ \\
\hline Salsolinol & & 34 & $14,98,99$ & $14,89,92-98,100-110$ \\
\hline 1,3-Dimethyl-TIQ & & 64,65 & - & 111 \\
\hline
\end{tabular}




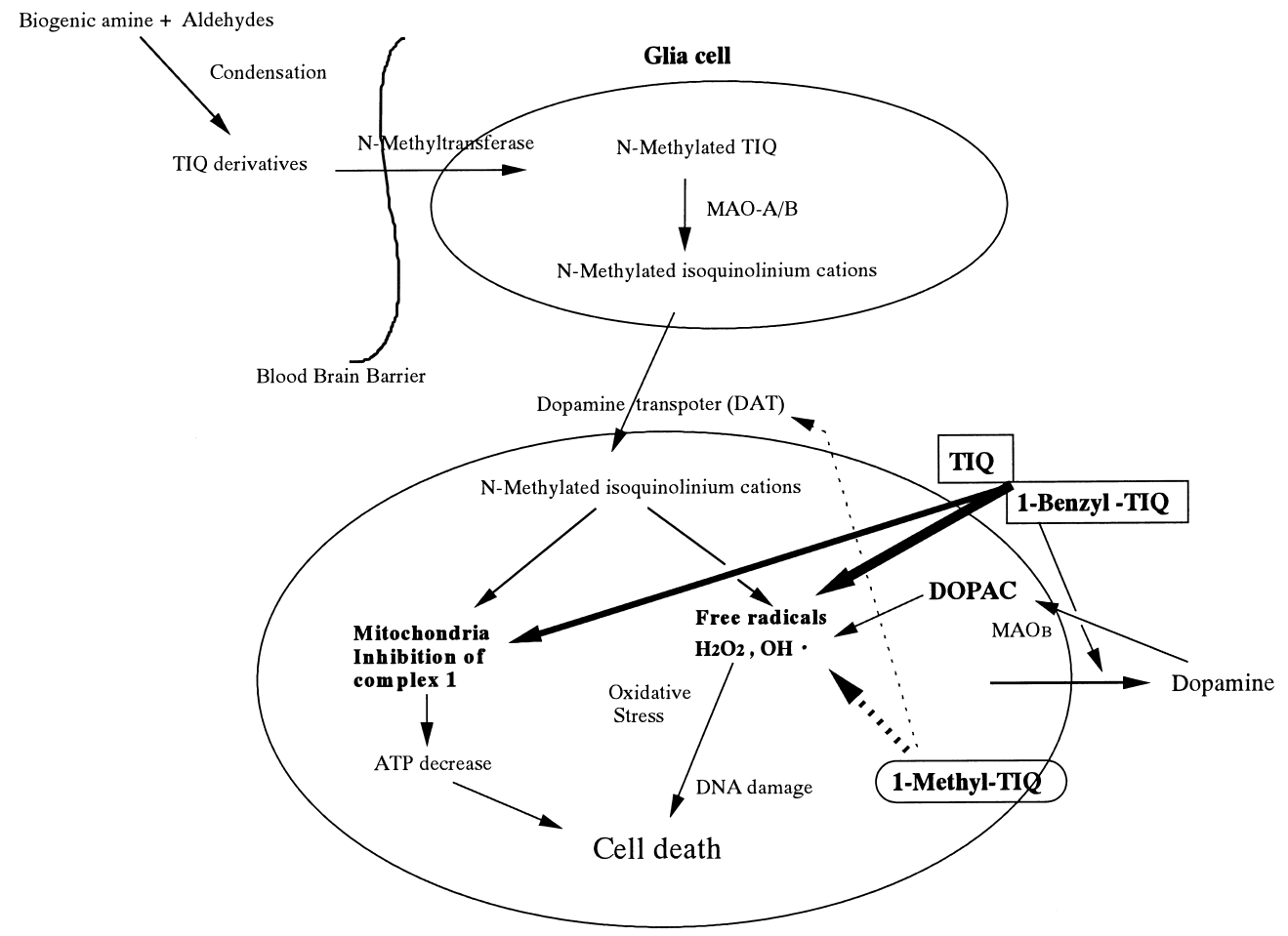

Dopamine neuron

Fig. 1. Hypothetical Mechanism of TIQ Neurotoxicity and Neuroprotection

TIQ, injected peripherally, crosses the blood brain-barrier and is transformed by glial MAO into the active compound isoquinolinium cation. The isoquinolinium cation crosses the neuronal membrane by a specific uptake mechanism. Once inside the dopaminergic neurons, isoquinolinium cations lead to an inhibition of the respiratory chain and free radical formation, both triggering cell death. Bold line indicates facilitation. Dashed line indicates inhibition.

tives-induced neurotoxicity and neuroprotection (Fig. 1).

\section{CONCLUSIONS}

Considerable progress has been made during the past 20 years in better understanding the active site of TIQ derivatives for PD at the pharmacological, biochemical and physiological levels. The data do not clearly identify the exact dopaminergic neuronal death involved in PD models. However, common mechanisms exist in dopaminergic neuronal death induced in humans or after exposure to neurotoxic TIQ derivatives. Throughout this review, we presented evidence suggesting that experimental models using TIQ and its derivatives reproduce the main mechanism of dopaminergic neuronal death occurring in PD. TIQ derivative neurotoxicity depends on a preferred accumulation site in the brain, the speed of synthesis, and propensity to form free radicals. Although not fully understood, the rate of synthesis and long-term exposure to endogenous TIQ derivatives in the brain could be a key factor in the progressive degeneration of the nigrostriatal dopaminergic system. Therefore, the continuous interaction between TIQ derivative-induced experimental models and human neuropathology will undoubtedly help to determine the crucial factors in dopaminergic neuronal death. In addition, investigations of the synthesis and biological properties of TIQ derivatives will be useful for the development of new therapeutic agents for treating PD.

\section{REFERENCES}

1) Imai Y., Soda M., Inoue H., Hattori N., Mizuno Y., Takahashi R., Cell, 105, 891-902 (2001).

2) Shimura H., Schlossmacher M. G., Hattori N., Frosch M. P., Trockenbacher A., Schneider R., Mizuno Y., Kosik K. S., Selkoe D. J., Science, 293, 263-269 (2001).

3) Langston J. W., Ballard P., Tetrud J. W., Irwin I., Science, 219, 979 980 (1983).

4) Davis P. A., Baird-Lambert J., Taylor K. M., Maclaren J. A., Biochem. Pharmacol., 28, 1803-1806 (1979).

5) Langston J. W., Forno L. S., Rebert C. S., Irwin I., Brain Res., 292, 390-394 (1984).

6) Schneider J. S., Yuwiler A., Markham C. H., Exp. Neurol., 91, 293 307 (1986).

7) Jarvis M. F., Wagner G. C., Life Sci., 36, 249-254 (1985).

8) Heikkila R. E., Sieber B. A., Manzino L., Sonsalla P. K., Mol. Chem. Neuropathol., 10, 171-183 (1989).

9) Hao R., Norgren R. B., Jr., Lau Y. S., Pfeiffer R. F., Neurology, 45, 138 - 142 (1995).

10) Hao R., Ebadi M., Pfeiffer R. F., Neurosci. Lett., 200, 77-80 (1995).

11) Deitrich R., Erwin V., Annu. Rev. Pharmacol. Toxicol., 20, 55-80 (1980).

12) Naoi M., Matsuura S., Takahashi T., Nagatsu T., Biochem. Biophys. Res. Commun., 161, 1213-1219 (1989).

13) Naoi M., Matsuura S., Parvez H., Takahashi T., Hirata Y., Minami M., Nagatsu T., J. Neurochem., 52, 653-655 (1989).

14) McNaught K. S., Carrupt P. A., Altomare C., Cellamare S., Carotti A., Testa B., Jenner P., Marsden C. D., Biochem. Pharmacol., 56, 921933 (1998).

15) Naoi M., Dostert P., Yoshida M., Nagatsu T., Adv. Neurol., 60, 212 217 (1993).

16) Naoi M., Maruyama W., Eur. Neurol., 33 (Suppl. 1), 31-37 (1993).

17) Niwa T., Takeda N., Kaneda N., Hashizume Y., Nagatsu T., Biochem. Biophys. Res. Commun., 144, 1084-1089 (1987).

18) Niwa T., Takeda N., Sasaoka T., Kaneda N., Hashizume Y., Yoshizumi 
H., Tatematsu A., Nagatsu T., J. Chromatogr., 491, 397-403 (1989).

19) Kotake Y., Tasaki Y., Makino Y., Ohta S., Hirobe M., J. Neurochem., 65, 2633-2638 (1995).

20) Matsubara K., Kobayashi S., Kobayashi Y., Yamashita K., Koide H., Hatta M., Iwamoto K., Tanaka O., Kimura K., Neurology, 45, 2240 2245 (1995).

21) Yamakawa T., Ohta S., Biochem. Biophys. Res. Commun., 236, 676681 (1997).

22) Kohno M., Ohta S., Hirobe M., Biochem. Biophys. Res. Commun., 140, 448-454 (1986).

23) Nakagawa H., Nihonmatsu N., Ohta S., Hirobe M., Biochem. Biophys. Res. Commun., 225, 1027-1034 (1996).

24) Tasaki Y., Makino Y., Ohta S., Hirobe M., J. Neurochem., 57, 19401943 (1991).

25) Yoshida M., Niwa T., Nagatsu T., Neurosci. Lett., 119, 109-113 (1990).

26) Makino Y., Ohta S., Tachikawa O., Hirobe M., Life Sci., 43, 373-378 (1988).

27) Niwa T., Yoshizumi H., Tatematsu A., Matsuura S., Nagatsu T., J. Chromatogr., 493, 347-352 (1989).

28) Kikuchi K., Nagatsu Y., Makino Y., Mashino T., Ohta S., Hirobe M., Drug Metab. Dispos., 19, 257-262 (1991).

29) Niwa T., Takeda N., Tatematsu A., Matsuura S., Yoshida M., Nagatsu T., J. Chromatogr., 452, 85-91 (1988).

30) Pictet A., Spengler T., Ber., 44, 2030-2036 (1911).

31) Bischler A., Napieralski B., Ber, 26, 1903-1908 (1893).

32) Pomeranz C., Monatsh. Chem., 14, 116-119 (1893).

33) Fritsch P., Ber., 26, 419-428 (1893).

34) Graig P. N., Nabenhauer F. P., Williams P. M., Macko E., Toner J., $J$. Am. Chem. Soc., 74, 1316-1317 (1952).

35) Venugopalan B., Brossi A., Heterocycles, 25, 359-364 (1987).

36) Christiff J. J., Bradley L., Miller D. D., Lei L., Rodrigez F., Fraundorfer P., Romstedt K., Shams G., Feller D. R., J. Medicinal Chem., 40, 8591 (1997).

37) Bates H. A., Bagherir K., Vertino P. M., J. Org. Chem., 51, 30613063 (1986).

38) Venkov A. P., Lukanov L. K., Synthetic Commun., 26, 755-762 (1996).

39) Kametani T., Fukumoto K., Aguri H., Yagi H., Kigasawa K., Sugahara H., Hiiragi M., Hyasaka T., Ishimaru H., J. Chem. Soc. Section C, 1998, 112-118 (1998).

40) Kametani T., Shibuya S., Satoh M., Chem. Pharm. Bull., 16, 953-957 (1968).

41) Kametani T., Kigasawa K. Hiiragi M., Ishimaru H., J. Chem. Soc. Section C, 1971, 2632-2634 (1971).

42) Scully F. E., Jr., Schlager J. J., Heterocycles, 19, 653-656 (1982).

43) Seebach D., Lohmann J.-J., Syfrig M. A., Yoshifuji M., Tetrahedron, 39, 1963-1974 (1983).

44) Kessar S. V., Sihgh P., Vohra R., Kaur N. P., Sihgh K. N., J. Chem. Soc., Chem. Commun., 1991, 568-570 (1991).

45) Kessar S. V., Vohra R., Kaur N. P., Sihgh K. N., Sihgh P., J. Chem. Soc., Chem. Commun., 1994, 1327-1328 (1994).

46) Heer J. P., Harling J. D., Thompson M., Synthetic Commun., 32, $2555-2563$ (2001).

47) Yokoyama A., Ohwada T., Shudo K., J. Org. Chem., 64, 611-617 (1999).

48) Takano S., Iida H., Inomata K., Ogasawara K., Heterocycles, 35, 4752 (1993).

49) Shinohara T., Toda J., Sano T., Chem. Pharm. Bull., 45, 813-819 (1997).

50) Shinohara T., Takeda A., Toda J., Sano T., Heterocycles, 46, 555-565 (1997).

51) Shinohara T., Takeda A., Toda J., Ueda Y., Kohono M., Sano T., Chem. Pharm. Bull., 46, 918-917 (1998).

52) Meyers A. I., Fuentes L. M., Kubota Y., Tetrahedron, 40, 1361-1370 (1984).

53) Meyers A. I., Boes M., Dickman D. A, Angew. Chem., 96, 448- 449 (1984).

54) Kotake Y., Yoshida M., Ogawa M., Tasaki Y., Hirobe M., Ohta S., Neurosci. Lett., 217, 69-71 (1996).

55) Makino Y., Tasaki Y., Ohta S., Hirobe M., Biomed. Environ. Mass Spectrom., 19, 415-419 (1990).

56) Ohta S., Kohno M., Makino Y., Tachikawa O., Hirobe M., Biomed. Res., 8, 453-456 (1987).

57) Ayala A., Parrado J., Cano J., Machado A., Brain Res., 638, 334-336
(1994).

58) Rein K., Goicoechea-Pappas M., Anklekar T. V., Hart G. C., Smith G. A., Gawley R. E., J. Am. Chem. Soc., 111, 2211-2217 (1989).

59) Yamato M., Hashigaki K., Qais N., Ishikawa S., Tetrahedron, 46, 5909-5920 (1990).

60) Hashigaki K., Kan K., Qais N., Takeuchi Y., Yamato M., Chem. Pharm. Bull., 39, 1126-1131 (1991).

61) Adam S., Pannecoucke X., Comberet J.-C., Quirion J.-C., J. Org. Chem., 66, $8744-8750$ (2001).

62) Ludwing M., Polborn K., Wanner K. T., Heterocycles, 61, 299-326 (2003).

63) Shinohara T., Takeda A., Toda J., Sano T., Chem. Pharm. Bull., 46, $430-433$ (1998).

64) Toda J., Matsumoto S., Saitoh T., Sano T., Chem. Pharm. Bull., 48, 91-98 (2000).

65) Saitoh T., Shikiya K., Horiguchi Y., Sano T., Chem. Pharm. Bull., 51, $667-672$ (2003)

66) Antkiewicz-Michaluk L., Romanska I., Papla I., Michaluk J., Bakalarz M., Vetulani J., Krygowska-Wajs A., Szczudlik A., Neuroscience, 96, 59-64 (2000).

67) Lorenc-Koci E., Smialowska M., Antkiewicz-Michaluk L., Golembiowska K., Bajkowska M., Wolfarth S., Neuroscience, 95, 10491059 (2000).

68) Nagatsu T., Yoshida M., Neurosci. Lett., 87, 178-182 (1988).

69) Ogawa M., Araki M., Nagatsu I., Nagatsu T., Yoshida M., Biogenic Amines, 6, 427-436 (1989).

70) Booth R. G., Trevor A., Singer T. P., Castagnoli N., Jr., J. Med. Chem., 32, 473- 477 (1989).

71) Abe K., Taguchi K., Wasai T., Ren J., Utsunomiya I., Shinohara T., Miyatake T., Sano T., Brain Res. Bull., 56, 55-60 (2001).

72) Ishiwata K., Koyanagi Y., Abe K., Kawamura K., Taguchi K., Saitoh T., Toda J., Senda M., Sano T., J. Neurochem., 79, 868-876 (2001).

73) Ishiwata K., Koyanagi Y., Abe K., Kawamura K., Taguchi K., Saitoh T., Toda J., Sano T., Brain Res., 960, 282-285 (2003).

74) Storch A., Schwarz J., "Neorotoxic Factors in Parkinson's Disease and Related Disorders," ed. by Storch A., Collins M. A., Plenum Press, New York, 2000, pp. 17-40.

75) Uhl G. R., Ann. Neurol., 43, 555-560 (1998).

76) Dunnett S. B., Björklund A., Nature (London), 399 (Suppl.), A32-39 (1999).

77) Lang A. E., Lozano A. M., N. Engl. J. Med., 339, 1030-1053 (1998).

78) Suzuki K., Mizuno Y., Yoshida M., Neurosci. Lett., 86, 105-108 (1988).

79) Suzuki K., Mizuno Y., Yoshida M., Neurochem. Res., 15, 705-710 (1990).

80) Parrado J., Absi E., Ayala A., Castano A., Cano J., Machado A., J. Neurochem., 75, 65-71 (2000).

81) Ricaurte G. A., Langston J. W., DeLanney L. E., Irwin I., Brooks J. D., Neurosci. Lett., 59, 259-264 (1985).

82) Antkiewicz-Michaluk L., Michaluk J., Mokrosz M., Romanska I., Lorenc-Koci E., Ohta S., Vetulani J., J. Neurochem., 78, 100-108 (2001).

83) Kotake Y., Taguchi R., Okuda K., Sekiya K., Tasaki Y., Hirobe M., Ohta S., Brain Res., 1033, 143-150 (2005).

84) Kotake Y., Tasaki Y., Hirobe M., Ohta S., Brain Res., 787, 341-343 (1998).

85) Gray N. M., Cheng B. K., Mick S. J., Lair C. M., Contreras P. C., J. Med. Chem., 32, 1242-1248 (1989).

86) Kotake Y., Ohta S., Kanazawa I., Sakurai M., Neuroscience, 117, 63 70 (2003).

87) Abe K., Taguchi K., Wasai T., Ren J., Utsunomiya I., Shinohara T., Miyatake T., Sano T., Brain Res., 907, 134-138 (2001).

88) Ishiwata K., Koyanagi Y., Saitoh T., Taguchi K., Toda J., Sano T., Senda M., J. Neural Transm., 108, 1111-1125 (2001).

89) Morikawa N., Naoi M., Maruyama W., Ohta S., Kotake Y., Kawai H., Niwa T., Dostert P., Mizuno Y., J. Neural. Transm., 105, 677—688 (1998).

90) Mizuno Y., Sone N., Saitoh T., J. Neurochem., 48, 1787-1793 (1987).

91) Shavali S., Ebadi M., Neurotoxicology, 24, 417-424 (2003).

92) Storch A., Ott S., Hwang Y. I., Ortmann R., Hein A., Frenzel S., Matsubara K., Ohta S., Wolf H. U., Schwarz J., Biochem. Pharmacol., 63, 909-920 (2002).

93) Okuda K., Kotake Y., Ohta S., Bioorg. Med. Chem. Lett., 13, 28532855 (2003). 